\title{
An Analytical Model for GMPLS Control Plane Resilience Quantification
}

\author{
Marc Ruiz, Jordi Perelló, Luis Velasco, Salvatore Spadaro, and Jaume Comellas
}

\begin{abstract}
This paper concentrates on the resilience of the Generalized Multi-Protocol Label Switching (GMPLS) enabled control plane. To this end, the problem of control plane resilience in GMPLS-controlled networks is firstly stated and previous work on the topic reviewed. Next, analytical formulae to quantify the resilience of generic meshed control plane topologies are derived. The resulting model is validated by simulation results on several reference network scenarios.
\end{abstract}

Index Terms-GMPLS, modeling, resilience.

\section{INTRODUCTION}

$\mathbf{T}$ HE vast majority of works on network resilience target at the transport plane. First and foremost, given the nowadays ultra-high transmission rates, milliseconds' failure recovery times may easily lead to terabit data losses. Besides, as the control information has been typically transmitted along with the data traffic (e.g., as in IP or MPLS networks), both control and data planes are equally affected upon failures, which makes no sense to separate both planes resilience.

However, in-band control plane configuration is not feasible in all-optical networks, as the end-to-end connections optically bypass all intermediate nodes from source to destination. In view of this, a separation is introduced in GMPLS [1] between the control and data planes, so that the control plane information can be transmitted on a different wavelength of the same fiber (in-fiber out-of-band) or even on a separated network (out-of-fiber). Thus, the reliability of the control plane in GMPLS-controlled networks becomes no more linked with the one of the data plane.

This provides several benefits to network operators, but new challenges are also posed to provide the control plane with the requirements to fulfil necessities of emerging services. Among the main benefits, there is an enhanced flexibility in the control deployment or the possibility to design control-plane-driven data plane recovery mechanisms, especially for the out-of-fiber configuration, where the control plane remains alive upon data plane failures. Nonetheless, when the control plane becomes decoupled from the data plane, additional fault detection and recovery mechanisms are required for the former.

\section{Previous Work}

Only a few works have so far addressed the resilience of the GMPLS-enabled control plane. Amongst them, [2] and [3] highlighted the reasons of a decoupled control plane

Manuscript received July 24, 2009. The associate editor coordinating the review of this letter and approving it for publication was X. Cao.

The authors are with the Advanced Broadband Communications Center (CCABA), Universitat Politècnica de Catalunya (UPC), Barcelona, Spain (e-mail: \{mruiz, perello, lvelasco\}@ac.upc.edu, \{spadaro, comellas\}@tsc.upc.edu).

Digital Object Identifier 10.1109/LCOMM.2009.12.091550 in all-optical networks and addressed the new resilience requirements that this would impose. In addition, [4] and [5] concluded that the most severe GMPLS protocol disruptions due to message losses (random losses [4] or connectivity outages due to link failures [5]) were found in RSVP-TE [1].

Comparing the approaches in [4] and [5], it seems more reasonable to have bursty message losses due to link connectivity outages, rather than random losses due to, e.g., network congestion. In fact, the load in the GMPLS control plane (i.e., RSVP-TE+OSPF-TE+LMP messages [1]) should not be very large under normal network operation (connection arrivals in the seconds' or minutes' time scales).

In order to evaluate the resilience of a given control plane topology, this work also focuses on the consequences of the control link failures on a GMPLS-controlled network performance, since these are the most probable ones in transport networks [6]. To this aim, the authors in [5] proposed a parameter $P_{d}$ that stands for the probability that any connection request or tear-down is dropped along the failure recovery time $\Delta t$ (i.e., forwarded onto the failed control link). Both situations would affect the network Grade Of Service (GoS), by either blocking/delaying a connection request, or keeping allocated but not used data plane resources.

An analytical $P_{d}$ formulation in symmetrical ring control planes was presented in [5]. As will be reviewed in section III, the final $P_{d}$ expression depends on the incoming (Poisson) traffic characteristics $(\lambda, \mu), \Delta t$, and $P_{L}$, which denotes the probability that an incoming connection request/tear-down is supported on the failed control link.

Even though ring networks have been extensively deployed over the years, operators are currently moving to deploy meshed network architectures, offering richer connectivity and, thus, enhanced survivability [6]. Therefore, it would be highly desirable to have tools for quantifying the control plane resilience in such scenarios. This letter aims at attempting, for the first time, an analytical formulation to quantify the resilience of generic asymmetrical [2] out-of-fiber meshed control plane configurations. Specifically, this will be obtained by extending the work for symmetrical ring control planes previously presented [5], broadening its scope to larger, more practical and more reliable control plane topologies.

\section{Analytical Model}

Equation (1) reproduces the analytical $P_{d}$ expression obtained in [5], where Poisson traffic arrivals to the network were assumed. In this expression, $C \approx\lceil\lambda / \mu\rceil$ identifies the number of active connections in the network at the failure time.

$$
P_{d}=1-e^{-\lambda \Delta t\left(1+P_{L}\right)} \sum_{k=0}^{C}\left(\begin{array}{l}
C \\
k
\end{array}\right)\left[\left(e^{\mu \Delta t}-1\right)\left(1-P_{L}\right)\right]^{k} .
$$


Note that the mathematical analysis behind $P_{d}$ is valid to any network scenario, as it basically depends on the traffic characteristics. The parameter that captures the network topology under study (and the traffic distribution over it) is $P_{L}$, which was particularized for symmetrical ring topologies in [5]. This section targets at a general $P_{L}$ expression to allow $P_{d}$ computation in asymmetrical meshed control planes.

Let $G_{D P}\left(N_{D P}, E_{D P}\right)$ and $G_{C P}\left(N_{C P}, E_{C P}\right)$ identify the data and control plane graphs of a GMPLS-enabled transport network, respectively. For the ongoing model we assume that $G_{D P}$ is bi-connected and planar. In fact, $G_{D P}$ topology can be seen as a set of interconnected sub-rings, that for highly meshed networks can be as small as triangles. We also assume $G_{C P}$ bi-connected, providing survivability to the control plane. Particularly, we restrict the control plane topology to be a subset (or the complete set) of the data plane one. Thus, $G_{D P}$ and $G_{C P}$ can be related as:

$$
\begin{gathered}
N_{C P} \equiv N_{D P} \equiv N \\
E_{C P} \subseteq E_{D P} .
\end{gathered}
$$

In this scenario, we define a minimal bi-connected covering topology over $G_{D P}$ (e.g. a Hamiltonian cycle or a minimum $n$-tree), so that $E_{D P}^{i t}$ identifies the link subset in this minimal topology and $E_{D P}^{o t}$ the subset containing the rest of the data plane links. Hence, $E_{D P} \equiv E_{D P}^{i t}+E_{D P}^{o t}$. In what follows, this additional relation between $G_{D P}$ and $G_{C P}$ is imposed:

$$
E_{C P} \supseteq E_{D P}^{i t}
$$

A minimal control plane topology $\left(E_{C P} \equiv E_{D P}^{i t}\right)$ is defined. On this basis, any intermediate topology (hereafter, partially meshed) is created by adding links to the minimal topology, finally getting the symmetrical topology $\left(E_{C P} \equiv E_{D P}\right)$.

From the assumptions above, $G_{C P}$ consists at least on one ring. Every link in $E_{D P}^{o t}$ added to $E_{C P}$ creates a new sub-ring, either by sub-ring partitioning (splitting an existing sub-ring in two) or sub-tree closing (adding a new sub-ring external to the minimal topology). In any case, two data plane adjacent nodes will belong to the same sub-ring at the control plane.

Let us define $H_{D P}$ as the average hop length of the data paths. In a similar way, $H_{C P}$ defines the average hop length of the control paths. As the RSVP-TE messages forwarded on the control plane should visit (i.e., configure) the same node sequence comprised in the computed data plane route, $H_{C P}$ becomes a function of $G_{D P}$ and $G_{C P}$.

At this point, we can define $P_{L}=D_{L} / D_{T}$, that is, the ratio between the amount of demands supported in the failed link $L\left(D_{L}\right)$ with respect to the total number of demands $\left(D_{T}\right)$. This finally leads to

$$
P_{L}=\frac{C \cdot H_{C P} /\left|E_{C P}\right|}{C}=\frac{H_{C P}}{\left|E_{C P}\right|} .
$$

As shown, $P_{L}$ directly depends on the average hop length of control plane paths. As mentioned above, end-to-end RSVPTE messages are processed hop-by-hop at every node in the route of the Label Switched Path (LSP) being signalled/torndown. As a consequence of equation (3), adjacent nodes in the data plane may be not adjacent in the control plane. Thus, $H_{C P}$ is proportional to $H_{D P}$, and can be expressed as

$$
H_{C P}=\tau \cdot H_{D P}
$$

where the parameter $\tau$ adjusts the distance (the number of hops) in the control plane between two adjacent nodes in the data plane.

Without loss of generality, we consider that every demand is routed through the shortest path. Besides, as in [5], we assume the traffic uniformly distributed in the network. Then, the average length of the shortest paths in a mesh network can be approximated by [7]:

$$
H_{D P} \approx \sqrt{\frac{|N|-2}{\delta_{D P}-1}}
$$

where $\delta_{D P}$ is the average node degree in the data plane.

To calculate $\tau$ we compute the distance at the control plane of all adjacent node pairs at the data plane. Being also adjacent at the control plane their distance equals to 1 . Otherwise, their distance in the control plane $\left(h_{j}^{G_{C P}}\right)$ is computed. Finally, $\tau$ can be expressed as

$$
\begin{aligned}
& \tau=\left(\sum_{\forall i \in E_{C P}} 1+\sum_{\forall j \in E_{D P} \backslash E_{C P}} h_{j}^{G_{C P}}\right) \frac{1}{\left|E_{D P}\right|}=\frac{\left|E_{C P}\right|}{\left|E_{D P}\right|}+ \\
& +\frac{\left|E_{D P}\right|-\left|E_{C P}\right|}{\left|E_{D P}\right|} \cdot \frac{\sum_{j \in E_{D P} \backslash E_{C P}} h_{j}^{G_{C P}}}{\left|E_{D P}\right|-\left|E_{C P}\right|}=\alpha+(1-\alpha) \cdot \kappa
\end{aligned}
$$

where $\alpha$ is the proportion of links at the control plane to those at the data plane, and $\kappa$ represents the average distance of non-adjacent nodes at the control plane.

We have focused on a minimal $G_{C P}$ topology consisting on a Hamiltonian cycle, where the average lengths of $E_{D P}^{o t}$ and $E_{D P}^{i t}$ links is similar. There, we have concluded (after several tests) that $\kappa$ can be accurately estimated as $\sqrt{|N|}$. In a more general case, every sub-ring in the control plane acts as a cycle covering a subset of nodes of $G_{D P}$. Based on the previous results, we approximate $\kappa \approx \sqrt{V_{C P}}$, where $V_{C P}$ is the mean number of nodes in a sub-ring.

As mentioned before, every pair of adjacent nodes at the data plane belongs to the same sub-ring at the control plane. Let $R_{C P}$ denote the number of sub-rings at the control plane, and $T_{C P}$ the sum of nodes in every individual sub-ring. Thus, $V_{C P}$ satisfies

$$
V_{C P}=\left\lceil\frac{T_{C P}}{R_{C P}}\right\rceil
$$

where

$$
\begin{gathered}
T_{C P} \approx\left|E_{D P}^{i t}\right|+2 \cdot\left|E_{D P}^{o t}\right|=2 \cdot\left|E_{C P}\right|-\left|E_{D P}^{i t}\right| \\
R_{C P}=\left|E_{C P}\right|-|N|+1 .
\end{gathered}
$$

Note that equation (10) gives an exact $T_{C P}$ value when all sub-rings have been created by sub-ring partitioning. In any other case, however, it still represents a valid approximation, 

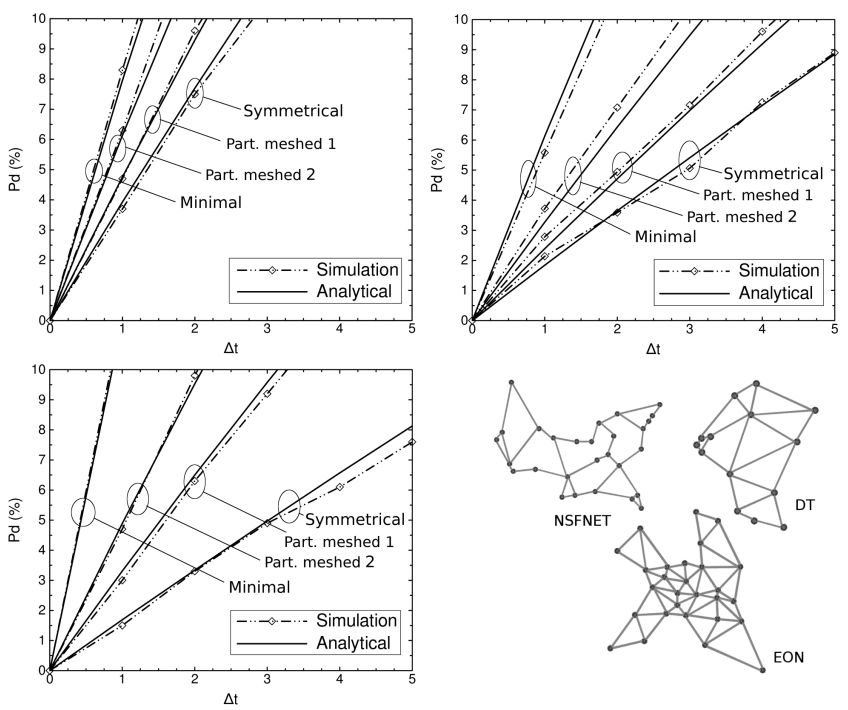

Fig. 1. Model vs. simulation results: NSFNET (top left); DT (top right); EON (bottom). All simulations are conducted under the same offered traffic to the network.

since sub-ring partitioning is much more frequent than subtree closing. Finally, combining equations (6), (7), (8) and (9), $P_{L}$ can be stated as

$$
P_{L} \cong\left[\alpha+(1-\alpha) \cdot \sqrt{V_{C P}}\right] \cdot \sqrt{\frac{|N|-2}{\delta_{D P}-1}} \cdot \frac{1}{\left|E_{C P}\right|}
$$

\section{Model Validation And Discussion}

The obtained $P_{d}$ model has been validated over different networks with different average node degrees. To this end, we consider a quite sparse 28-Node NSFNET topology, a moderately meshed 14-Node Deutsche Telekom (DT) network, and a highly meshed 28-Node European Optical Network (EON). Besides, for each topology, we define four different control plane alternatives: a symmetrical topology, a minimal topology, and two partially meshed topologies in between. Table I reviews the most relevant parameters of each topology under evaluation. The column on the right presents $\left|E_{C P}\right|$ in the symmetrical, partially meshed 1, partially meshed 2 and minimal topologies, respectively.

The performance of the model has been validated by simulation results. For them, enough wavelengths per link to guarantee that all requests are routed through the shortest path (accomplishing the wavelength continuity constraint) are assumed. In such scenarios, uniformly distributed connection requests arrive at each node following a Poisson process, and connection holding times are exponentially distributed. The model and the simulation results for $P_{d}$ as a function of $\Delta t$ are plotted in Fig. 1. Each simulation is conducted in order to reach steady state results within a $95 \%$ confidence interval.

As seen, the $P_{d}$ model and the simulation results are really close in every experimented topology. Aiming to measure the discrepancy between the obtained $P_{d}$ values and the expected ones, we have computed the Chi-square goodness of fit test in each scenario. To this goal, we compare the number of affected connections obtained by simulation with respect to
TABLE I

NETWORK TOPOLOGY PARAMETERS

\begin{tabular}{|c|c|c|c|c|}
\cline { 2 - 5 } \multicolumn{1}{c|}{} & $|N|$ & $\left|E_{D P}\right|$ & $\delta_{D P}$ & $\left|E_{C P}\right|$ \\
\hline NSFNET & 28 & 37 & 2.64 & $37-34-31-29$ \\
\hline DT & 14 & 23 & 3.28 & $23-20-17-14$ \\
\hline EON & 28 & 61 & 4.36 & $61-41-34-28$ \\
\hline
\end{tabular}

the expected value of this variable (i.e., multiplying the $P_{d}$ analytical value by the number of total simulated connections). In all cases, the null hypothesis can be clearly accepted (the difference between simulation and analytical results is zero), which highlights the accuracy of the model.

Motivated by the necessity of quality of resilience parameters, $P_{d}$ could be proposed to quantify the maximum recovery time to meet certain control plane resilience requirements (i.e., a certain $P_{d}$ value). In particular, the minimal topology requires very restrictive $\Delta t$ values (Fig. 1). Since multiple demands are supported on each control link, the performance degradation caused by control link failures is very high. For instance, aiming at a $P_{d}=5 \%$ objective in the 28-Node EON, $\Delta t<500 \mathrm{~ms}$ must be assured. However, by increasing the connectivity at the control plane, $P_{d}$ steadily decreases. In the symmetrical topology, as only one demand is supported on each control link, $\Delta t \approx 3 \mathrm{~s}$ already fits $P_{d}=5 \%$. Between both extremes we have the partially meshed topologies, which target at a trade-off between resilience and required resources.

Network operators could benefit from the proposed model to quantify the number of control plane links needed to fit certain $P_{d}$ requirements, given a $\Delta t$ achievable by their control plane recovery mechanisms (e.g., IP layer re-routing, dedicated link protection...). This value could be afterwards used as input data for an optimal control plane topology design.

\section{ACKNOWLEDGMENT}

The work described in this paper was carried out with the support of the BONE Project ("Building the Future Optical Network in Europe"), a Network of Excellence funded by the European Commission through the 7th ICT-Framework Programme. Moreover, it was supported by the Spanish science ministry through the project ENGINE (TEC2008-02634).

\section{REFERENCES}

[1] E. Mannie, "Generalized multi-protocol label switching architecture," IETF RFC 3945, Oct. 2004.

[2] A. Jajszczyk and P. Rozyki, "Recovery of the control plane after failures in ASON/GMPLS networks," IEEE Network, vol. 20, no. 1, Jan. 2006.

[3] G. Li, J. Yates, D. Wang, and C. Kalmanek, "Control plane design for reliable optical networks," IEEE Commun. Mag., vol. 40, no. 2, Feb. 2002.

[4] O. Komolafe and J. Sventek, "Impact of GMPLS control message loss," IEEE/OSA J. Lightwave Technol., vol. 26, no. 14, July 2008.

[5] J. Perelló, S. Spadaro, J. Comellas, and G. Junyent, "An analytical study of control plane failures impact on GMPLS ring optical networks," IEEE Commun. Lett., vol. 11, no. 8, Aug. 2007.

[6] W. Grover, Mesh-Based Survivable Transport Networks: Options and Strategies for Optical, MPLS, SONET and ATM Networking. Prentice Hall, 2003.

[7] S. K. Korotky, "Network global expectation model: a statistical formalism for quickly quantifying network needs and costs," IEEE/OSA J. Lightwave Technol., vol. 22, no. 3, Mar. 2004. 